\title{
MAGNETIC FIELDS IN OH MASER CLOUDS
}

\author{
R. D. DAVIES \\ University of Manchester, Nuffield Radio Astronomy Laboratories, \\ Jodrell Bank, Cheshire, United Kingdom
}

\begin{abstract}
Observations of Class I OH maser sources show a range of features which are predicted on the basis of Zeeman splitting in a source magnetic field. Magnetic field strengths of 2 to $7 \mathrm{mG}$ are derived for eight $\mathrm{OH}$ maser sources. The fields in all the clouds are directed in the sense of galactic rotation. A model of W3 $\mathrm{OH}$ is proposed which incorporates the magnetic field data. It is shown that no large amount of magnetic flux or angular momentum has been lost since the condensation from the interstellar medium began.
\end{abstract}

\section{Introduction}

Compact $\mathrm{OH}$ maser sources show strong polarization - especially those associated with $\mathrm{H}$ II regions (Class I OH sources). A characteristic of these sources is their strong (nearly $100 \%$ ) circular polarization and weaker linear polarization. When circular polarization was first detected, it was attributed to Zeeman splitting by magnetic fields of a few milligauss in the $\mathrm{OH}$ cloud (Davies et al., 1966). Subsequently it became clear that these sources were masering and this simple explanation was not sufficient, and other explanations were sought.

Shklovskii (1969) suggested that twin source components of opposite circular polarization might be produced by a rotating $\mathrm{OH}$ cloud in which the lines are split by a Zeeman effect. His method of producing circular polarization is similar to that suggested earlier by Cook (1966).

Linear polarization can result from directional pumping by unpolarised radiation which produces irregularities in the Zeeman sublevel populations, even in the absence of significant magnetic fields (e.g., Perkins et al., 1966). The more frequently observed circular polarization cannot be formed in this way.

Proposals for generating circular polarization have been advanced (Heer and Graft, 1965; Culshaw and Kanneland, 1966; Heer and Settles, 1967) which are based upon saturated masers where one of the senses of polarization is suppressed. These theories predict patterns of emission and polarization for the other lines of ${ }^{2} \Pi_{3 / 2}$, $J=3 / 2$ quartet which are not confirmed by observations (Litvak, 1969; Turner, 1970). The suppression theory has been criticized by Bender (1967), who showed that the likelihood of producing a resultant circular polarization was much less than had been calculated. Further, the Heer and Settles theory required a magnetic field of $\lesssim 10^{-10} \mathrm{G}$, a value considerably less than any likely interstellar value. In a similar theory Hall and ter Haar (1973) have recently discussed the circular and linear polarization resulting from saturated maser mode competition occurring in a single-line model of Class I $\mathrm{OH}$ sources.

The possibility of a Zeeman interpretation of the circular polarization from some $\mathrm{OH}$ maser sources has again found favour with some recent authors (Coles et al., 
1969; Rydbeck et al., 1970; Gardner et al., 1970; Litvak, 1971; and Zuckerman et al., 1972). Goldreich et al. (1973) have given a theoretical discussion of the role of Zeeman splitting in producing polarization in astrophysical masers. Further it is recognized that in an interstellar maser there is unlikely to be a strict one-to-one correspondence between LH and RH components (Davies, 1967).

The present paper examines the Zeeman splitting interpretation in some detail and shows that for a number of sources the predictions are verified by observations of single telescope spectra for a wide range of transitions and also by interferometer data. Magnetic field strengths are derived for a number of $\mathrm{OH}$ maser sources, and these are compared with the values expected for the compression of the interstellar field. The dynamical stability of the $\mathrm{OH}$ sources is also discussed.

\section{The Expected Zeeman Splitting of the OH Molecule}

(a) THE ZEEMAN PATTERN

In this section the normal Zeeman pattern of $\mathrm{OH}$ will be described for the various transitions which have been observed in $\mathrm{OH}$ maser sources. These include the ${ }^{2} \Pi_{1 / 2}$, $J=1 / 2$ state triplet, $J=3 / 2$ and $5 / 2$ quartets and the ${ }^{2} \Pi_{3 / 2}, J=3 / 2$ and $5 / 2$ quartets.

Each of the $\Pi$ ground electron states mentioned above is split into two states by $\Lambda$ doubling, and these in turn are doubled by hyperfine splitting, resulting in four states for each value of $J$. In a magnetic field each of these states is further split into $2 F+1$ magnetic energy sublevels separated from the original energy level by

$$
\begin{aligned}
\Delta W=h \Delta v= & -\mu_{n} g_{I} \cdot \frac{M_{F} B}{2 F(F+1)} \cdot[I(I+1)+F(F+1)-J(J+1)], \\
& -\mu_{0} g_{J} \cdot \frac{M_{F} B}{2 F(F+1)} \cdot[J(J+1)+F(F+1)-I(I+1)],
\end{aligned}
$$

where $\mu_{n}$ and $\mu_{0}$ are the nuclear and Bohr magneton $\left(\mu_{n}=\mu_{0} / 1836\right)$

$g_{1} \sim g_{J} \sim 1$ (see below for actual values of interest);

$\mu_{0} / h=1.39967 \mathrm{MHz} \mathrm{G}^{-1}$;

$J=$ quantum number for the total angular momentum including rotation but excluding nuclear spin;

$F=$ total angular momentum;

$I=$ nuclear spin;

$M_{F}=$ the magnetic quantum number giving the projection of $F$ on the direction of the magnetic field; it takes the values $F, F-1 \ldots-F+1, F$;

$B=$ the magnetic field strength.

For the ${ }^{2} \Pi_{3 / 2}$ states of $\mathrm{OH}$ the first (nuclear and magnetic moment) component of the splitting is negligible compared with the second (electronic) component. However, in the ${ }^{2} \Pi_{1 / 2}$ states the spin and orbital components of the electron magnetic moment are oppositely directed and largely cancel, and the nuclear spin component is a significant contribution; the resultant splitting is $\sim 10^{-3}$ of the ${ }^{2} \Pi_{3 / 2}$ states. 
The Zeeman splitting pattern expected for a particular $\mathrm{OH}$ line results from transitions between the magnetic sublevels of the two states. Allowed transitions are $\Delta M_{F}=0$ ( $\pi$ components) and $\Delta M_{F}= \pm 1$ ( $\sigma$ components). The resulting Zeeman patterns for the ${ }^{2} \Pi_{3 / 2}, J=3 / 2$ and $5 / 2$ states are shown in Figure 1. When the field is directed parallel to the line of sight the $\mathrm{LH}$ and $\mathrm{RH} \sigma$ components are seen; when
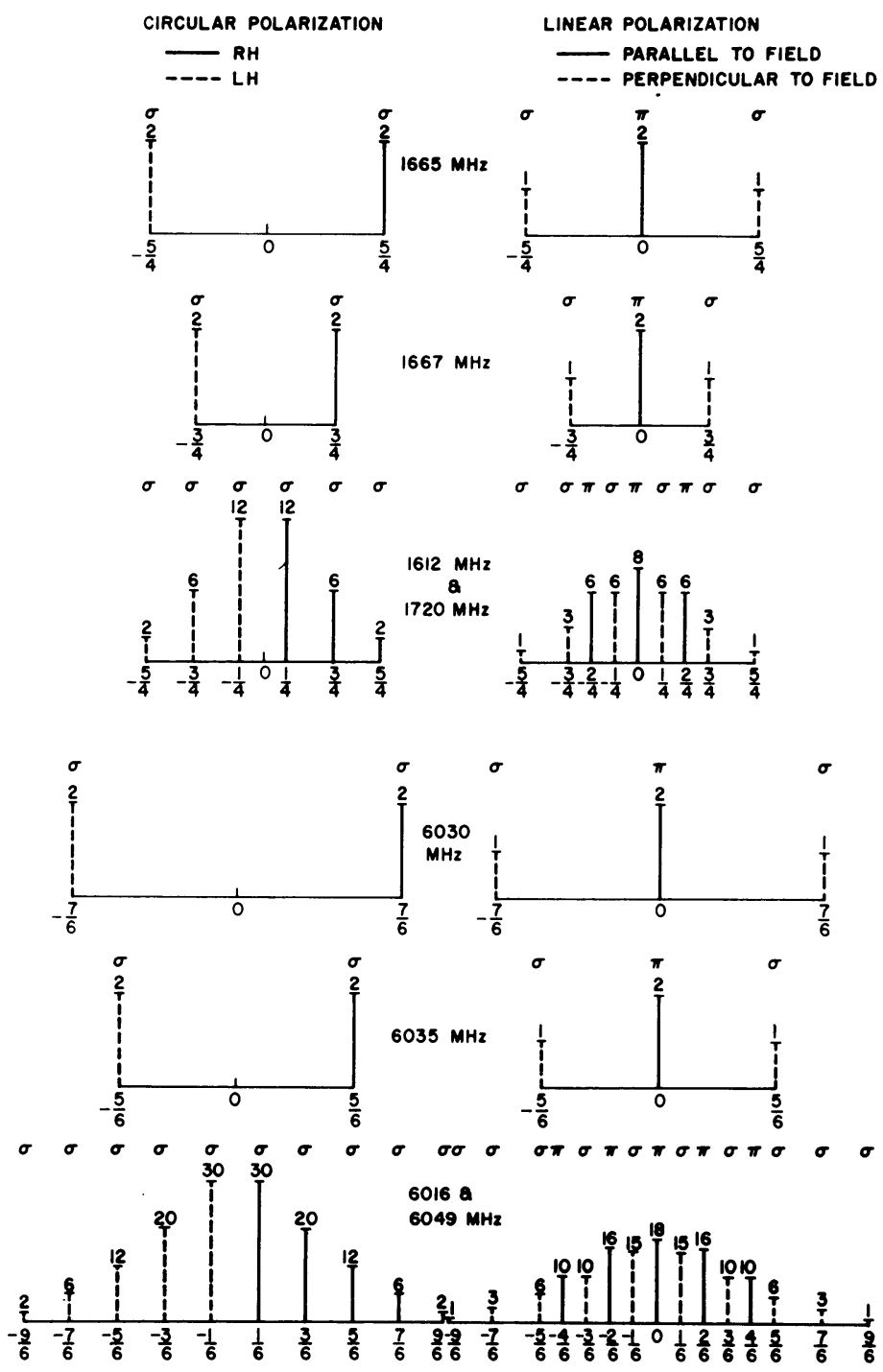

Fig. 1. Zeeman splitting patterns for the ${ }^{2} \Pi_{3 / 2}, J=3 / 2$ and $J=5 / 2$ quartets of $O H$. The frequency splitting is plotted in units of $g_{J} . \mu_{0} / h$; this is $1.31 \mathrm{MHz} \mathrm{G}^{-1}$ for the $J=3 / 2$ lines and $0.679 \mathrm{MHz} \mathrm{G}^{-1}$ for the $J=5 / 2$ lines. Relative intensities of the Zeeman components are shown for each transition. The patterns in the left-hand side of the figure result from observations parallel to the field and those on the right-hand side are from observations perpendicular to the field. 
perpendicular to the line of sight the $\pi$ components are linearly polarized parallel to the field while the $\sigma$ components are perpendicular. The intensities of the various $\sigma$ and $\pi$ components have been estimated using Table 10.1 of Townes and Schawlow (1956); relative intensity values are given in Figure 1.

The amount of Zeeman splitting in the commonly observed $\mathrm{OH}$ transitions is given in Table I. These refer to the $\sigma$ components and have been estimated from the values of $g_{J}(=0.935,0.485$ and 0.325 for $J=3 / 2,5 / 2$ and $7 / 2)$ for the ${ }^{2} \Pi_{3 / 2}$ state given by Radford (1961). In the case of the satellite lines which have many $\sigma$ components, the tabulated Zeeman split is the average split weighted by the component intensity. Table I also emphasizes the smaller Zeeman splitting measured in terms of velocity for the higher frequency excited hydrogen lines.

(b) APPLICATION TO AN OH MASERING SOURCE

In any astronomical situation the polarized components shown in Figure 1 will be broadened by thermal and mass motions of the $\mathrm{OH}$ molecules. When the broadening is comparable to the Zeeman splitting the observed polarization will be reduced. A typical cloud is illustrated in Figure 2, where the emission spectra on a given line of sight show the effects of differing mass motions and magnetic fields; the field direction is assumed to have a component away from the observer at each point. If the $\mathrm{OH}$ were not masering and it had a constant excitation temperature its emission spectrum (proportional to $\int n_{\mathrm{OH}}(v) \mathrm{d} l$ ) in each polarization might appear as in (a). However, if the molecular energy states were inverted so that the emission column was an unsaturated maser the resultant spectra might look like (b), where each spectrum will be proportional to $\exp \left[\right.$ const $\left.\times \int n_{\mathrm{OH}}(v) \mathrm{d} l\right]$. In (a) there are only small fractional differences in the LH and RH spectra, whereas in (b) these differences have been ampli- . fied by the exponential term to give strongly polarized signals. For example, in the

TABLE I

Zeeman splitting of $\mathrm{OH}$ lines ( $\sigma$ components)

\begin{tabular}{|c|c|c|c|c|}
\hline \multirow[t]{2}{*}{ Transition } & & \multirow{2}{*}{$\begin{array}{l}\text { Frequency } \\
(\mathrm{MHz})\end{array}$} & \multicolumn{2}{|c|}{ Zeeman split between $\mathrm{LH}$ and $\mathrm{RH}$} \\
\hline & & & $\left(\mathrm{MHz} \mathrm{G}^{-1}\right)$ & $\left(\mathrm{km} \mathrm{s}^{-1} \mathrm{G}^{-1}\right)$ \\
\hline${ }^{2} \Pi_{1 / 2} J=1 / 2$ & $\begin{aligned} F= & 0 \rightarrow 1 \\
& 1 \rightarrow 1 \\
& 1 \rightarrow 0\end{aligned}$ & $\begin{array}{l}4660.2 \\
4750.6 \\
4765.5\end{array}$ & $\begin{array}{l}\sim 10^{-3} \\
\sim 10^{-3} \\
\sim 10^{-3}\end{array}$ & $\begin{array}{l}\sim 10^{-2} \\
\sim 10^{-2} \\
\sim 10^{-2}\end{array}$ \\
\hline${ }^{2} \Pi_{3 / 2} J=3 / 2$ & $\begin{aligned} F=1 & \rightarrow 2 \\
1 & \rightarrow 1 \\
2 & \rightarrow 2 \\
2 & \rightarrow 1\end{aligned}$ & $\begin{array}{l}1612.2 \\
1665.4 \\
1667.3 \\
1720.5\end{array}$ & $\begin{array}{l}1.308 \\
3.270 \\
1.964 \\
1.308\end{array}$ & $\begin{array}{l}236 \\
590 \\
354 \\
236\end{array}$ \\
\hline$J=5 / 2$ & $\begin{aligned} F=2 & \rightarrow 3 \\
& \rightarrow \rightarrow 2 \\
3 & \rightarrow 3 \\
3 & \rightarrow 2\end{aligned}$ & $\begin{array}{l}6016.7 \\
6030.7 \\
6035.0 \\
6049.0\end{array}$ & $\begin{array}{l}0.678 \\
1.582 \\
1.132 \\
0.678\end{array}$ & $\begin{array}{l}33.8 \\
79.0 \\
56.4 \\
33.8\end{array}$ \\
\hline
\end{tabular}




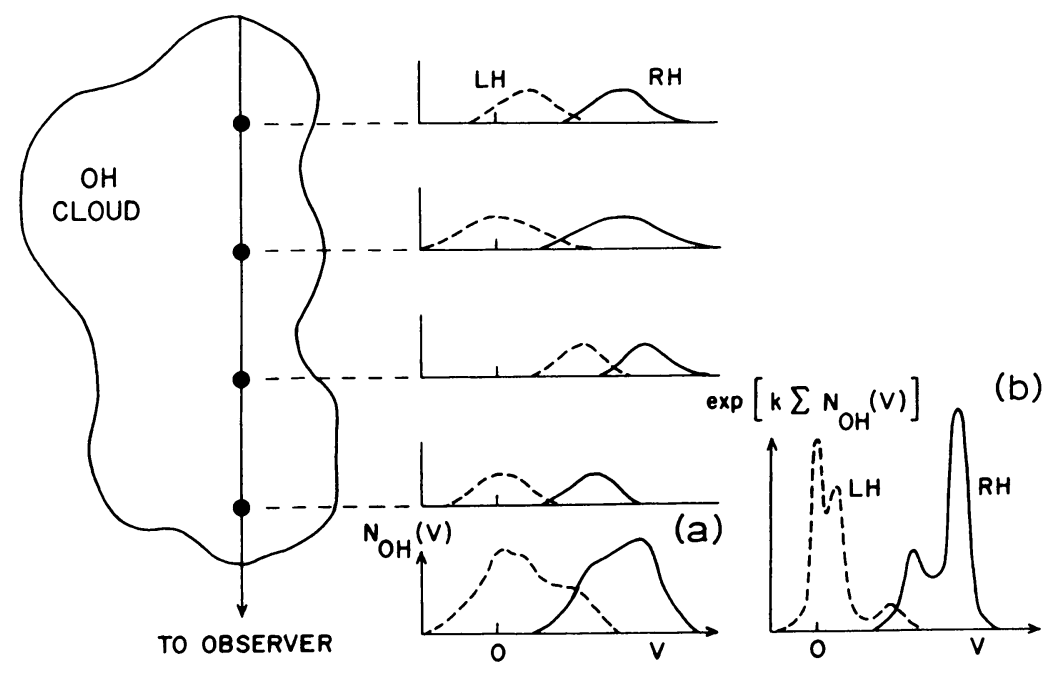

Fig. 2. The masered spectrum produced by contributions along a line of sight through an $\mathrm{OH}$ cloud. The distribution of molecules as a function of velocity, $N_{\mathrm{OH}}(v)$, at each point is shown. The sum of all contributions to $N_{\mathrm{OH}}$ is $\Sigma N_{\mathrm{OH}}(v)$. The resultant masered spectrum $T_{B}(v)$ is of the form $\exp$ [const $\times \Sigma N_{\mathrm{OH}}(v)$ ]. The two circularly polarized components are shown by full and broken lines.

stronger $\mathrm{OH}$ sources the exponent is $20-30$, so a fractional difference of 0.2 in the $\mathrm{LH}$ and RH line integral spectra would give a difference of $e^{4}-e^{6}$ (i.e., 50-400) in the masered LH and RH spectra. Moreover, the $\mathrm{LH}$ and RH integrals are not identical, nor are they frequency-shifted versions of one another; accordingly the LH and RH masered spectra are in general very different. In addition the pumping may produce different amounts of inversion along the line of sight, and this will enhance the differences between the $\mathrm{LH}$ and $\mathrm{RH}$ spectra.

If the Zeeman splitting is comparable with (say $20 \%$ or more) the total Doppler broadening of a source, a high percentage of circular polarization of the masered spectrum is expected. The amount of linear polarization in this situation will be less than the circular polarization both in terms of the number of masered lines and their percentage polarization. This is evident from Figure 1, where the orthogonal linear components have only half the frequency separation of the circular components, and, further, the outer linear components have the same polarization. Further, Faraday depolarization along the emission column and propagation path in the source can reduce or remove linear polarization but will not affect circular polarization.

The above statements, based on a superposition approach, are essentially justified by Goldreich et al. (1973), who have shown in a detailed discussion of the transfer equations for the Stokes parameters that there is no polarization unless the Zeeman splitting exceeds the bandwidth of the amplified radiation when the emitted radiation is just the amplified Zeeman pattern. If the Faraday rotation across the region is large they show that the linear polarization is destroyed while the $\sigma$ components of the Zeeman pattern are $100 \%$ circularly polarized. 
TABLE II

Zeeman splitting measurements in $\mathrm{OH}$ sources

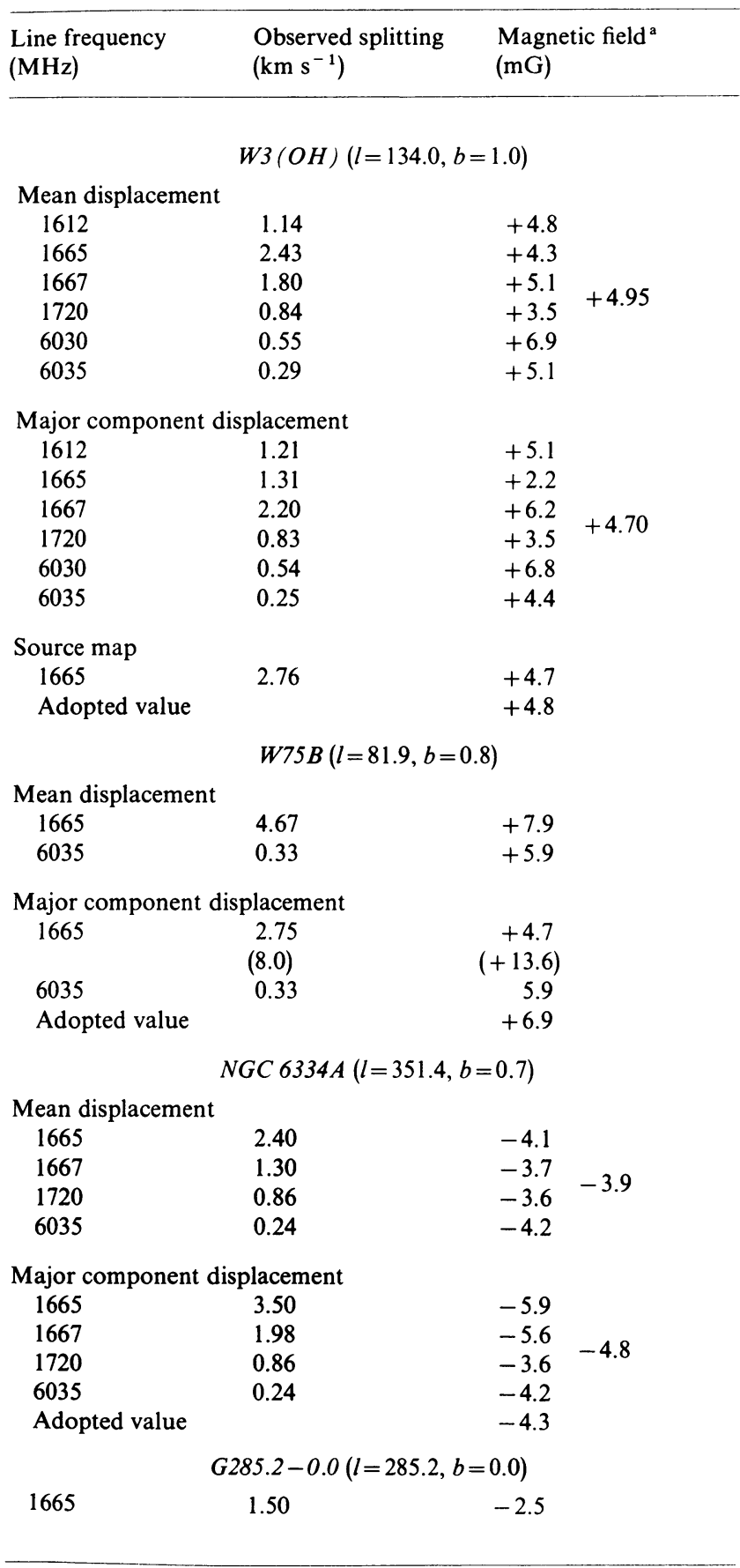


Table II (Continued)

\begin{tabular}{|c|c|c|c|}
\hline $\begin{array}{l}\text { Line frequency } \\
(\mathrm{MHz})\end{array}$ & $\begin{array}{l}\text { Observed splitting } \\
\left(\mathrm{km} \mathrm{s}^{-1}\right)\end{array}$ & $\begin{array}{l}\mathrm{Ma} \\
(\mathrm{mC}\end{array}$ & netic field ${ }^{a}$ \\
\hline \multicolumn{4}{|c|}{$G 291.6-0.4(l=291.6, b=-0.4)$} \\
\hline 1665 & 3.6 & -6.1 & \\
\hline \multicolumn{4}{|c|}{$N G C 6334 B(l=351.1, b=0.7)$} \\
\hline $\begin{array}{l}1665 \\
1667\end{array}$ & $\begin{array}{l}1.1 \\
0.8\end{array}$ & $\begin{array}{l}-1.9 \\
-2.3\end{array}$ & -2.2 \\
\hline \multicolumn{4}{|c|}{$\operatorname{Sgr} B 2(l=0.7, b=0.0)$} \\
\hline $\begin{array}{l}1665 \\
1720 \\
6035\end{array}$ & $\begin{array}{l}1.95 \\
0.90 \\
0.29\end{array}$ & $\begin{array}{l}+3.3 \\
+3.8 \\
+5.1\end{array}$ & +4.1 \\
\hline \multicolumn{4}{|c|}{$W 49(l=43.2, b=0.0)$} \\
\hline \multicolumn{4}{|l|}{$\begin{array}{l}\text { Interferometer } \\
1665\end{array}$} \\
\hline
\end{tabular}

a positive magnetic field is directed away from the observer.

(c) PREDICTIONS OF THE ZEEMAN SPLITTING EXPLANATION

The polarization properties of the maser emission from $\mathrm{OH}$ sources will now be summarized. It is supposed that the Zeeman splitting is comparable with the Doppler broadening for the lowest frequency lines $\left({ }^{2} \Pi_{3 / 2}, J=3 / 2\right)$ lines at $\sim 1660 \mathrm{MHz}$.

(i) A strict Zeeman pattern like Figure 1 is not expected for each emitting component for the reasons given above. If there is a dominant velocity component in the source the main polarized features (generally with unequal intensity) will be displaced by an amount given by the Zeeman split relation in Table I.

(ii) The masered spectra observed in different transitions will appear to have quite different component velocities because of the different ratio of Zeeman to Doppler shift in the various transitions.

(iii) Circular polarization will dominate the spectra. The number of linear components and their percentage polarization will be small because they overlap.

(iv) All the ${ }^{2} \Pi_{3 / 2}$ lines will show the same sense of overall displacement between $\mathrm{RH}$ and $\mathrm{LH}$ spectra provided there is a consistent field direction in the cloud.

(v) The mean velocity displacement of the LH and RH spectra will approximate the values listed in Table I and will be greatest for the 1665 and $1667 \mathrm{MHz}$ transitions.

(vi) The Zeeman component of the velocity displacement of the LH and RH spectra in ${ }^{2} \Pi_{3 / 2}, J=5 / 2$ transitions will be a factor of $\sim 7$ less than in the $J=3 / 2$ transitions.

(vii) Transitions in the ${ }^{2} \Pi_{1 / 2}$ state will be unpolarized.

(viii) Interferometer observations will show the $\mathrm{LH}$ and $\mathrm{RH}$ emission components from the same general area of the source (i.e., having the same systematic velocity) with the appropriate Zeeman split for the field at that point. 


\section{The Zeeman Splitting Interpretation of Observed OH Spectra}

A number of $\mathrm{OH}$ maser sources of Class I have been studied in sufficient transitions to test the validity of the Zeeman interpretation. The magnetic fields in these sources will have to be sufficiently large that the Zeeman splitting in at least the 1665 and $1667 \mathrm{MHz}$ lines is comparable with the Doppler broadening. For a few brighter sources long baseline interferometry gives the relative spatial positions of the stronger LH and RH components; these can be compared with the predictions. Eight sources (W3 OH, W75 B, NGC $6334 A$ and $B$, G258.2-0.0, G291.6-0.4, Sgr B2 and W49) show some of the Zeeman effects described above.

\section{(a) $W 3(O H)\left(\right.$ R.A. $=02^{\mathrm{h}} 23^{\mathrm{m}} 17^{\mathrm{s}}$, Dec. $\left.=61^{\circ} 38^{\prime} 54^{\prime \prime}[1950]\right)$}

W3 is the brightest and the most extensively observed Class I OH maser source. Figure 3 plots the integrated LH and RH spectra of the stronger transitions taken from published material. They show all the characteristics (a) to (g) of Zeeman splitting discussed in Section IIc. Since there are approximately 20 components in the $1665 \mathrm{MHz}$ spectrum a statistical determination of the magnetic field responsible can be obtained from the mean displacement of the $\mathrm{LH}$ and $\mathrm{RH}$ spectra in the transitions. The velocity displacements for the six transitions and their corresponding magnetic fields are listed in Table III. These give a mean field of $4.95 \mathrm{mG}$ directed away from the observer. Another estimate of the magnetic field is provided by the displacement between the brightest $\mathrm{LH}$ and $\mathrm{RH}$ features in each spectrum. This gives a mean field of $4.7 \mathrm{mG}$. Although this method gives a similar value to the previous method, in general it is more liable to error because the brightest masered component is not necessarily representative of the mean spectrum.

Interferometry gives the third method of determining magnetic fields in an $\mathrm{OH}$ masering source by indicating directly the Zeeman displacement between the LH and RH components in each region of the source. $1665 \mathrm{MHz}$ observations of $\mathrm{W} 3 \mathrm{OH}$ by Harvey et al. (1973) give a mean field of $4.7 \mathrm{mG}$ in separated regions of the object. The three methods are consistent in indicating a field in $\mathrm{W} 3 \mathrm{OH}$ of $4.8 \mathrm{mG}$ directed away from the observer.

(b) $W 75 B$ (R.A. $=20^{\mathrm{h}} 36^{\mathrm{m}} 54^{\mathrm{s}}$, Dec. $\left.=42^{\circ} 25^{\prime} 40^{\prime \prime}[1950]\right)$

Observations at 1665 and $6035 \mathrm{MHz}$ of adequate sensitivity and frequency resolution are available for $\mathrm{W} 75 B(=\mathrm{W} 75 \mathrm{~N})$. The $6035 \mathrm{MHz}$ spectra show the same $\mathrm{LH}$ to RH displacement in the three major components. At $1665 \mathrm{MHz}$ it is not clear which major LH components should be combined with the two strongest RH components without reference to the $6035 \mathrm{MHz}$ splitting. Two splittings are suggested in Table II. The adopted field is $6.9 \mathrm{mG}$ directed away from the observer.

(c) NGC 6334A (R.A. $=17^{\mathrm{h}} 17^{\mathrm{m}} 32^{\mathrm{s}}$, Dec. $\left.=-35^{\circ} 44^{\prime} 15^{\prime \prime}[1950]\right)$

Figure 5 summarizes the published data on NGC $6334 H(=$ NGC $6334 N)$, which strongly supports the Zeeman interpretation. The splittings of four strong lines give a consistent field of $4.3 \mathrm{mG}$ directed towards the observer. 


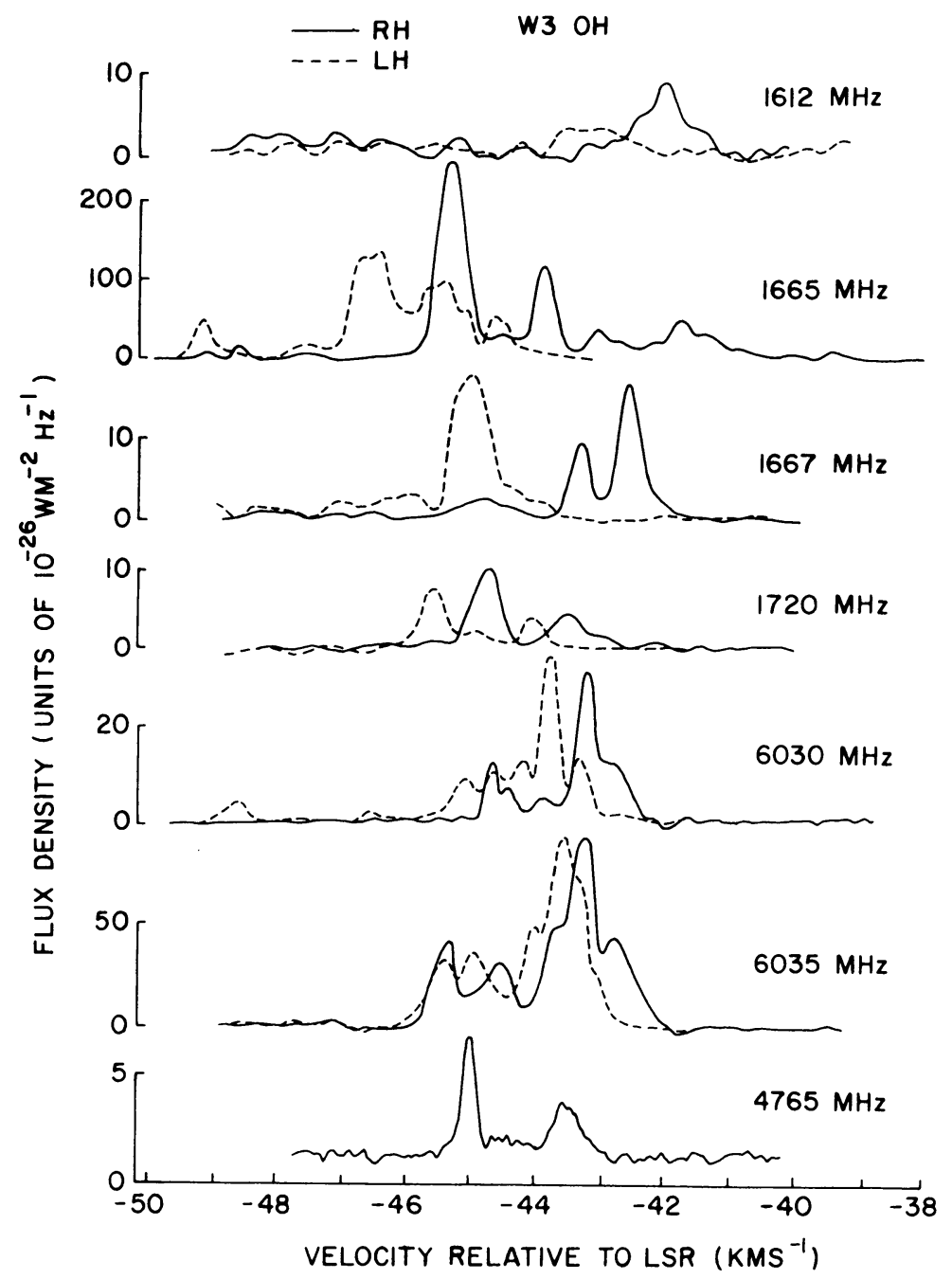

Fig. 3. Observed spectra of $\mathrm{W} 3 \mathrm{OH}$. RH polarization is shown by full lines and LH by broken lines. The $4765 \mathrm{MHz}$ spectrum is the sum of orthogonal polarizations; it is believed to be unpolarized.

(d) OTHER OH SOURCES

A number of other $\mathrm{OH}$ sources have been observed with adequate frequency resolution and show spectral line displacements that indicate Zeeman splitting. The clearest examples are G285.2-0.0, G291.6-0.4, NGC 6334B and Sgr B2. The LH and RH displacements and the derived magnetic field strengths and directions are given in Table II. Of these sources Sgr B2 is the most complex; consistent splittings in three transitions are suggested. Another source, W49, shows strong frequency splitting of the $\mathrm{LH}$ and RH components in many of its sub-sources when mapped at $1665 \mathrm{MHz}$ with an interferometer (Harvey et al., 1973). The corresponding fields in the five sub- 


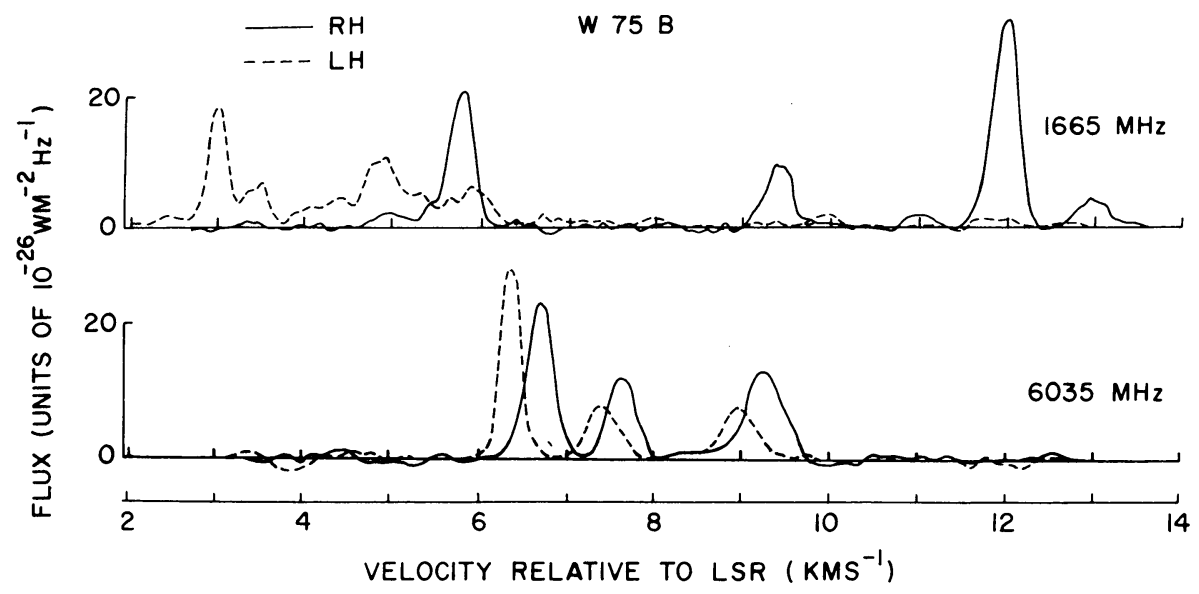

Fig. 4. Observed spectra of $\mathrm{W} 75 B(=\mathrm{W} 75 N)$. RH polarization is shown by full lines and LH by broken lines.

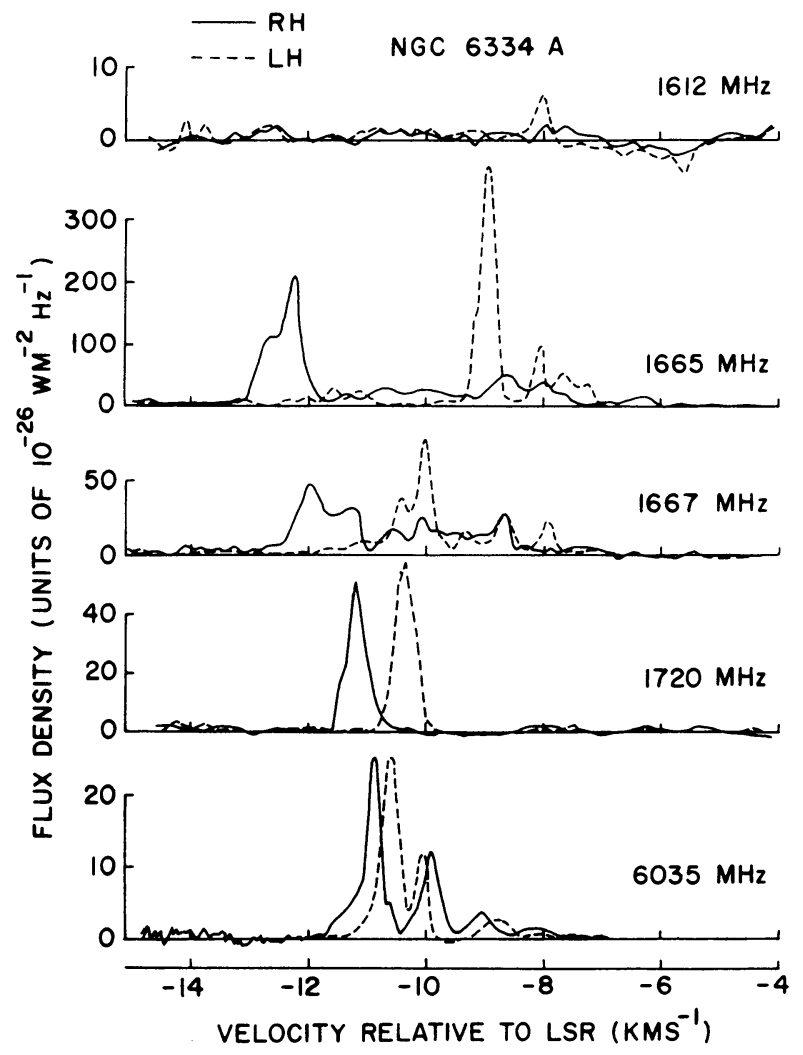

Fig. 5. Observed spectra of NGC $6334 A$. RH polarization is shown by full lines and LH by broken lines. 
sources of W49 range from 0.7 to $5.2 \mathrm{mG}$ directed away from the observer in each case.

\section{Magnetic Field Amplification in Interstellar Clouds}

Class I maser sources are closely associated with $\mathrm{H}$ II regions and areas of contemporary star formation. It is therefore to be expected that the $\mathrm{OH}$ masers are themselves gas clouds collapsing towards the protostar phase. It is quite possible that in many of these objects the central star is in the nuclear burning phase and has already ionized the gas in its immediate vicinity. This situation probably exists in $\mathrm{W} 3 \mathrm{OH}$ where an $\mathrm{H}$ II region and IR source are closely associated with the $\mathrm{OH}$ source. A number of similar associations are suspected. In this section we consider the effect of the collapse of the interstellar cloud on the magnetic field strength and alignment. Larson's (1969) models of collapsing protostars will be used.

If a uniform magnetic field $B_{0}$ permeates the average interstellar cloud with a hydrogen density $n_{0}(\mathrm{H})$ and the cloud is of such a configuration that it will collapse gravitationally then the field will also be compressed. Conservation of magnetic flux in an isotropic collapse will lead to a field $B$ at a density $n(\mathrm{H})$ given by

$$
B=B_{0}\left[\frac{n(\mathrm{H})}{n_{0}(\mathrm{H})}\right]^{2 / 3}
$$

Measurements of the Zeeman effect in normal interstellar H I clouds (Verschuur, 1971; Davies et al., 1974) show that this relationship is obeyed. The available $\mathrm{H}$ I data show that $B_{0}=4 \sqrt{ } 2 \mu \mathrm{G}$ for $n_{0}(\mathrm{H})=10 \mathrm{~cm}^{-3}$. For the $\mathrm{OH}$ maser sources estimates of $n(\mathrm{H})$ can be made from the measured $\mathrm{OH}$ maser gain which suggest that $n(\mathrm{H}) \sim 3 \times 10^{6}$ $\mathrm{cm}^{-3}$. The uncertainty in this is no more than a factor of 10 either way (see Section $\mathrm{V}$ for a discussion of $n(\mathrm{H})$ for $\mathrm{W} 3 \mathrm{OH}$ ). The corresponding value of $B$ would be $25 \mathrm{mG}$ if magnetic flux is conserved in an isotropic collapse. Reference to Table II shows that the highest observed values are only a factor of 4 less than this predicted magnetic field value.

An alternative estimate of the magnetic field amplification can be made by including the effect of the unimpeded infall along the magnetic axis of the cloud. If the radius in this direction is a fraction $\eta<1$ of the radius perpendicular to the field then the compressed field is $\eta^{2 / 3}$ of that calculated above. For $\eta=0.5$, a value consistent with the observed dimensions of $\mathrm{W} 3 \mathrm{OH}$, the value of $B$ would be reduced from 25 to $16 \mathrm{mG}$. In view of the uncertainty in estimating $n(\mathrm{H})$ it is clear that the observed values are consistent with magnetic flux conservation of the general interstellar field during gravitational cloud collapse. Only a small flux-loss is permitted by the data. It would therefore be surprising if the effects of Zeeman splitting were not detectable in those collapsing clouds which have reached a density of $\sim 10^{6} \mathrm{~cm}^{-3}$.

The relationship between $B$ and $n(\mathrm{H})$ given above will be preserved throughout the depth of the cloud even if the density varies with depth, providing the assumption of conservation of magnetic flux still holds. Thus in $\mathrm{W} 3 \mathrm{OH}$, where the 
OH lies within an outer shell surrounding ionized gas, we would expect the field to be still given by the expression since this gas is a part of the original gas cloud.

In the foregoing discussion it has been assumed that the magnetic field is frozen into the gas. This assumption can be justified on the following arguments. For a fully ionized gas the time for the Ohmic losses to dissipate an amount of energy comparable with the magnetic energy is given by Spitzer (1962) as

$$
\tau=2 \times 10^{-13} T^{2 / 3} L^{2} \text { seconds, }
$$

where $T$ is the gas temperature and $L$ is a length characteristic of the system. Putting $T=100 \mathrm{~K}$ and $L=10^{15} \mathrm{~cm}$, the smallest length scale found in the $\mathrm{OH}$ masers, $\tau=6 \times 10^{12} \mathrm{yr}$. This dissipation time far exceeds the collapse time of the cloud, which is $\sim 10^{5}$ yr (Larson, 1969). In a partially ionized gas, which is more appropriate for the $\mathrm{OH}$ cloud, Cowling (1957) gives a dissipation time of

$$
\tau_{B}=1.2 \times 10^{-3} n_{\mathrm{e}} n R^{2} B^{-2} \mathrm{yr}
$$

where $n_{\mathrm{e}}\left(\sim 10^{-4} n\right)$ and $n$ are in $\mathrm{cm}^{-3}, R$ the cloud radius in pc, and $B$ is in G. With a cloud radius of $0.012 \mathrm{pc}, n=10^{6} \mathrm{~cm}^{-3}$, we find $\tau_{B}=2 \times 10^{6} \mathrm{yr}$. This is still greater than the lifetime of the cloud. It is therefore evident that the magnetic field is frozen into the gas of the $\mathrm{OH}$ cloud.

A surprising pattern is found in the magnetic field directions within the eight $\mathrm{OH}$ masers listed in Table II. All the fields are aligned in the direction of galactic rotation, which is also the direction of the general galactic magnetic field determined from Faraday rotation measurements of pulsars and extragalactic sources (Gardner et al., 1967; Manchester, 1972). The neutral hydrogen clouds for which a significant Zeeman splitting has been measured also fit this pattern (Davies et al., 1974). The locations of the $\mathrm{OH}$ and $\mathrm{H}$ I clouds in the Galaxy are shown in Figure 6. This seems to indicate that these dense clouds which are widely spread throughout the Galaxy have preserved the direction of the general magnetic field as they have collapsed from densities of a few atoms per cubic centimeter to $\sim 10^{6} \mathrm{~cm}^{-3}$. The conditions under which such a cloud can collapse in diameter by a factor of $\sim 70$ and still preserve its alignment relative to the weak external field are of interest but will not be discussed further here.

It is pertinent to consider in more detail the implication that the Class I OH maser sources are associated with collapsing protostars. The number of $\mathrm{OH}$ maser sources would therefore be expected to equal the rate of formation of $\mathrm{O}$ and $\mathrm{B}$ stars multiplied by $10^{5} \mathrm{yr}$, the gravitational collapse time of the protostar; the $\mathrm{OH}$ maser action occurs only when the density is $\sim 3 \times 10^{6} \mathrm{~cm}^{-3}$. Estimates of the rate of formation of $\mathrm{O}$ stars in the Galaxy suggest $\sim 10^{-3} \mathrm{yr}^{-1}$ (Spitzer, 1968). This leads to an expected number of $10^{2} \mathrm{OH}$ sources in the Galaxy, a value which is consistent with the observations.

\section{A Model of the Magnetic Field in W3 OH}

In this section we will discuss a model of the magnetic field in the $\mathrm{W} 3 \mathrm{OH}$ maser source. This source has been observed extensively at a wide range of frequencies, and 


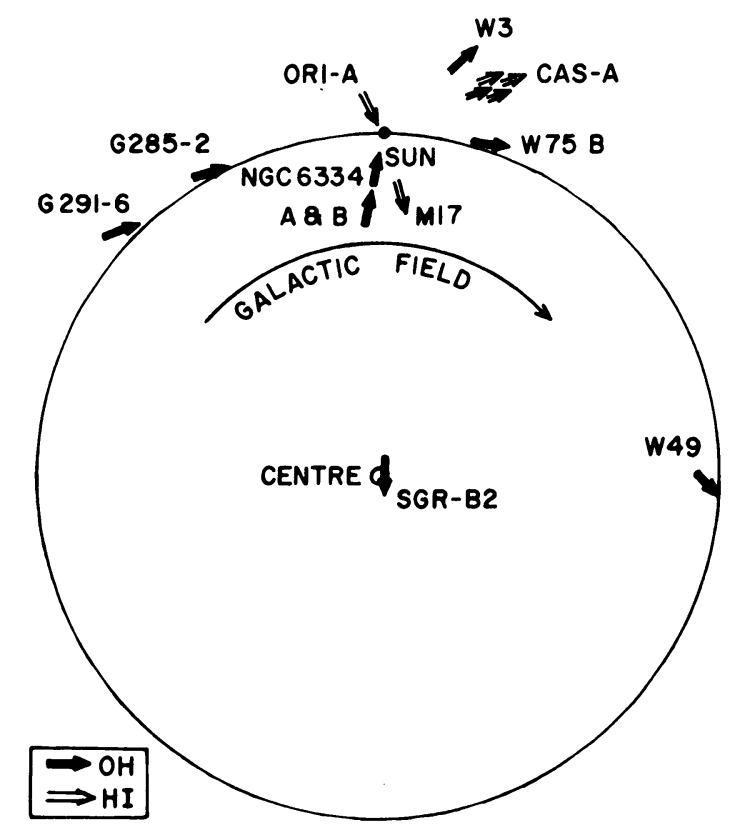

Fig. 6. The (line of sight) magnetic field direction in eight $\mathrm{OH}$ maser sources (this paper) and seven $\mathrm{H}_{\mathrm{I}}$ clouds (Davies et al., 1974). The general interstellar field of the Galaxy is directed in the clockwise sense. All the cloud fields are seen to be in the same sense.

it has been mapped in most detail. Its measured properties are as follows: (a) Its dimensions are $2.5^{\prime \prime} \times 1.4^{\prime \prime}(0.036 \times 0.020 \mathrm{pc})$ with a major axis at position angle $\sim 0^{\circ}$. (b) Its magnetic field is $4.8 \mathrm{mG}$. (c) When the effect of Zeeman splitting is allowed for in each region of the source a velocity gradient is found which indicates a rotational velocity of $\sim 2 \mathrm{~km} \mathrm{~s}^{-1}$ about an axis perpendicular to the major axis; the more negative velocities are at the northern end.

A sketch of the proposed model for $\mathrm{W} 3 \mathrm{OH}$ is shown in Figure 7. The magnetic field is taken to be parallel to the rotation axis for the following reason. The fields in the $\mathrm{OH}$ maser sources are found to be parallel to the general galactic field and consequently will be parallel to the galactic plane; for $\mathrm{W} 3 \mathrm{OH}$ this field will be directed to p.a. $\sim 270^{\circ}$. This is also the rotation axis inferred from the observations.

The W3 OH source is associated with an $\mathrm{H}$ II region with dimensions $2.3^{\prime \prime} \times 1.9^{\prime \prime}$ which is at the same position within the errors of measurements (Baldwin et al., 1973). An IR source is also associated; its dimensions are unknown. The rms density of the HII region is $1.25 \times 10^{5} \mathrm{~cm}^{-3}$. An $\mathrm{O} 7 \mathrm{star}$ at the centre of $\mathrm{W} 3 \mathrm{OH}$ is required to produce the ionization and to heat circumstellar dust to produce the IR emission (Wynn-Williams et al., 1972). The mass of the $\mathrm{O} 7 \mathrm{star}$ is $\sim 20 M_{\odot}$, while the $\mathrm{H}$ II region is $10^{-1} M_{\odot}$ and the $\mathrm{OH}$ cloud is $\sim 1.5 M_{\odot}$ (see below). The $\mathrm{OH}$ cloud, which also contains a magnetic field of $\sim 5 \times 10^{-3} \mathrm{G}$, is a cool shell surrounding the $\mathrm{H}$ II region. This outer gas which has a longer free-fall time is probably still collapsing inwards 


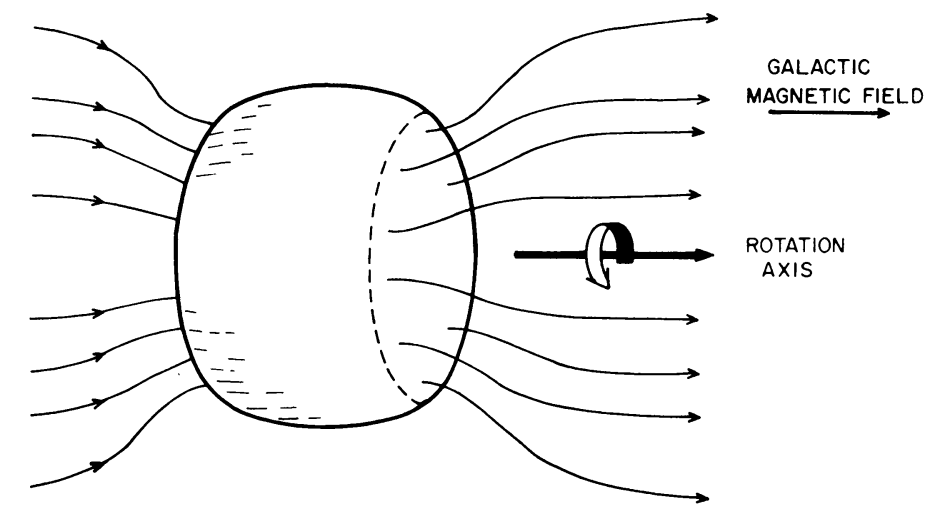

Fig. 7. A model of the $\mathrm{W} 3 \mathrm{OH}$ source showing the sense of rotation and the magnetic field direction. The $\mathrm{OH}$ source is a shell of gas surrounding an $\mathrm{H}$ II region and an $\mathrm{O} 7 \mathrm{star}$.

while the inner ionized gas is heated by the star formed from the greater mass of gas which collapsed first (Larson, 1969). The hot inner gas and the star are probably the source of the pumping mechanism of the Class I OH maser in $\mathrm{W} 3 \mathrm{OH}$.

An important parameter in this discussion is the gas density in the molecular masering cloud; this can be estimated from the observed maser gain. Various authors (e.g., Litvak, 1969; Litvak et al., 1969) have shown that the molecular line integral is given for a source similar to $\mathrm{W} 3 \mathrm{OH}$ by

$$
\int n(\mathrm{OH}) \mathrm{d} l \cdot \frac{v}{\Delta v} \approx 10^{22} \mathrm{~cm}^{-2},
$$

where $v / \Delta v \sim 3 \times 10^{5}$ is the ratio of the line frequency to the line-width before amplification. This gives $\int n(\mathrm{OH}) \mathrm{d} l=3 \times 10^{16} \mathrm{~cm}^{-2}$. With a masering depth of $\sim 10^{16} \mathrm{~cm}$ - a typical line of sight through the shell - we obtain $n(\mathrm{OH})=3 \mathrm{~cm}^{-3}$. Taking $n(\mathrm{H}) / n(\mathrm{OH})=10^{6}$ (Litvak, 1969) we find $n_{\mathrm{H}}=3 \times 10^{6} \mathrm{~cm}^{-3}$ with an uncertainty of a factor of 10 either way arising from uncertainties in the various steps of the calculation. This gas density would be in pressure equilibrium with the hot $\mathrm{H}$ II region gas if the temperature of the $\mathrm{OH}$ cloud were $\sim 10^{3} \mathrm{~K}$. The mass of a circumstellar gas cloud with this density and a thickness of $20 \%$ of the radius derived for the $\mathrm{OH}$ maser source would be $1.5 M_{\odot}$.

The dynamical stability of the $\mathrm{OH}$ maser source can be checked with the data available. If the collapse of the object were entirely stabilized by rotation a point on the equator would satisfy the relationship

$$
\frac{v^{2}}{R}=\frac{G M}{R^{2}}, \text { i.e., } v^{2}=\frac{G M}{R} .
$$

Using a total system mass $M \sim 20 M_{\odot}, R=4.5 \times 10^{16} \mathrm{~cm}$, the RH side of the equation leads to $v \sim 2.5 \mathrm{~km} \mathrm{~s}^{-1}$, whereas the observed rotational velocity is $2 \mathrm{~km} \mathrm{~s}^{-1}$. Thus the rotation is capable of largely stabilizing the source. In fact the flattening of the object may, in part, be due to the significant centrifugal force at the equator. 
The other likely contributor to the flattening of the source will be magnetic rigidity of the cloud in the plane perpendicular to the magnetic axis of the cloud. Estimates using the values of $n(\mathrm{H}), B$ and velocity spread $\left(1 \mathrm{~km} \mathrm{~s}^{-1}\right)$ given above show that the magnetic and gravitational pressures have a similar magnitude of $\sim 3$ to $10 \times 10^{-7}$ dyn $\mathrm{cm}^{-2}$ while the thermal gas pressure is about one tenth of this value. This magnetic pressure, acting orthogonally to the field direction will result in a significant flattening of the gas cloud parallel to the magnetic (and rotational) axis.

\section{Conclusion}

A study of the expected properties of Zeeman splitting of $\mathrm{OH}$ maser emisssion and a comparison with observed properties indicate that a number of the sources show all the properties expected for either saturated or unsaturated masers (Goldreich et al., 1973). These sources evidently have a Zeeman splitting comparable to or larger than the velocity dispersion of the $\mathrm{OH}$ gas. In other sources the spectra are so complex that even though the Zeeman effect is still producing the strong circular polarization it is impossible to decide which $\mathrm{LH}$ and $\mathrm{RH}$ components are related. Long baseline interferometry is shown to be an effective method of demonstrating the Zeeman splitting of such sources (e.g., W49). The main effects observed here are predicted in the models discussed by Goldreich et al. (1973), where the Zeeman splitting is greater than the (non-masered) line-width for unsaturated masers and for small Faraday rotation. Smaller fields than this could still produce strong circular polarization, but the Zeeman splitting patterns would be confused by random motions in the sources. It is interesting that the Class I OH maser sources have collapsed to such a density that the frozen-in magnetic fields are amplified so that they produce a Zeeman splitting comparable to the random motions and therefore give strong circular polarization.

In the sources where the field is sufficiently strong to separate the $\mathrm{LH}$ and $\mathrm{RH}$ circularly polarized components, estimates can be made of the magnetic field strength. These are in the range $2-7 \mathrm{mG}$. It is conjectured that the $\mathrm{OH}$ sources represent a much later phase of the collapse of the most dense clouds studied in neutral hydrogen Zeeman observations. The magnetic fields found in the $\mathrm{OH}$ clouds are of the magnitude expected for the conservation of magnetic flux. Only a small proportion, if any, of the magnetic flux appears to have been lost in collapsing from a density of atoms of $\sim 10 \mathrm{~cm}^{-3}$ to $\sim 3 \times 10^{6} \mathrm{~cm}^{-3}$. In a further isotropic collapse to stellar densities $\left(3 \times 10^{24} \mathrm{~cm}^{-3}\right)$ these fields would be amplified to $2 \times 10^{9} \mathrm{G}$; photospheric fields would be $\sim 10^{5} \mathrm{G}$. Since such high fields are not seen in normal stars, a mechanism is required for removing magnetic flux from the contracting cloud (see for example Spitzer, 1968). Most of this flux loss must therefore occur between $\mathrm{OH}$ maser densities and stellar densities, namely when the density is greater than $10^{6} \mathrm{~cm}^{-3}$ and the radius is less than $5 \times 10^{16} \mathrm{~cm}$. The magnetic field will be largely dissipated by hydromagnetic instabilities with a timescale equal to the dynamical collapse time of the cloud of $\sim 10^{5} \mathrm{yr}$ (Mestel, 1972). 
The effect of magnetic braking on the cloud angular momentum due to any coupling between the cloud magnetic field and the interstellar field can also be investigated for the $\mathrm{W} 3 \mathrm{OH}$ source. The present angular momentum per unit mass for the $\mathrm{OH}$ cloud as determined from its rotation velocity is $\sim 30 \%$ of the angular momentum of the cloud from which it collapsed if we take the interstellar turbulent velocity to be $0.1 \mathrm{~km} \mathrm{~s}^{-1}$ (Spitzer, 1968). Within the uncertainties of the parameters used there is no strong evidence for any large-scale loss of angular momentum. Like magnetic flux, the angular momentum loss occurs principally when the cloud collapses to higher densities than are represented by the $\mathrm{OH}$ sources. As a consequence the timescale for both loss processes must be $\lesssim 10^{5} \mathrm{yr}$, the collapse time of the $\mathrm{OH}$ cloud.

The recent measurements of magnetic fields in $\mathrm{HI}_{\mathrm{I}}$ and $\mathrm{OH}$ clouds are now providing a corpus of observational data which can be related to theoretical studies of the kind carried out by Mestel (1965) and Parker (1973) for example.

\section{Acknowledgements}

I wish to thank Professors F. D. Kahn and L. Mestel and Dr R. S. Booth for discussions on many of the topics of this paper.

\section{References}

Baldwin, J. E., Harris, C. S., and Ryle, M.: 1973, Nature 241, 38.

Bender, P. L.: 1967, Phys. Rev. Letters 18, 562.

Coles, W. A., Rumsey, V. H., and Welch, W. J. : 1968, Astrophys. J. Letters 154, L61.

Cook, A. H.: 1966, Nature 211, 503.

Cowling, T. G.: 1957, Magnetohydrodynamics, Interscience Publishers, New York, p. 110.

Culshaw, W. and Kanneland, J.: 1966, Phys. Rev. 145, 257.

Davies, R. D. : 1967, in H. van Woerden (ed.), 'Radio Astronomy and the Galactic System', IAU Symp. 31, 82.

Davies, R. D., de Jager, G., and Verschuur, G. : 1966, Nature 209, 974.

Davies, R. D., Booth, R. S., and Wilson, A. J.: 1974, in preparation.

Gardner, F. F., Ribes, J. C., and Goss, W. M.: 1970, Astrophys. Letters 7, 51.

Gardner, F. F., Whiteoak, J. B., and Morris, D.: 1967, Nature 214, 371

Goldreich, P., Keeley, D. A., and Kwan, J. Y.: 1973, Astrophys. J. 179, 111.

Hall, L. M. and ter Haar, D.: 1973, Monthly Notices Roy. Astron. Soc. 162, 97.

Harvey, P. J., Booth, R. S., Davies, R. D., Whittet, D., and McLaughlin, W.: 1974, Monthly Notices Roy. Astron. Soc., in press.

Heer, C. V. and Graft, R. G.: 1965, Phys. Rev. 140, A1088.

Heer, C. V. and Settles, R. A.: 1967, J. Mol. Spectrosc. 23, 448.

Larson, R. B.: 1969, Monthly Notices Roy. Astron. Soc. 145, 271.

Litvak, M. M.: 1969, Astrophys. J. 156, 471.

Litvak, M. M.: 1971, Astrophys. J. 170, 71.

Litvak, M. M., Zuckerman, B., and Dickinson, D. F.: 1969, Astrophys. J. 156, 875.

Manchester, R. N.: 1972, Astrophys. J. 172, 43.

Mestel, L.: 1965, Quart. J. Roy. Astron. Soc. 6, 161 and 265.

Mestel, L.: 1972, in P. Schindler (ed.), Cosmic Plasma Physics, Plenum Publishing Corporation, New York, p. 203.

Parker, D. A.: 1973, Monthly Notices Roy. Astron. Soc. 163, 41.

Perkins, F., Gold, T., and Salpeter, E. E.: 1966, Astrophys. J. 145, 361. 
Radford, H. E.: 1961, Phys. Rev. 122, 144.

Rydbeck, O. E. H., Kollberg, E., and Elldér, J. : 1970, Astrophys. J. Letters 161, L25.

Shklovskii, I. S.: 1969, Soviet Astron. 13, 1.

Spitzer, L.: 1962, Physics of Fully Ionized Gases, Interscience Publishers, New York.

Spitzer, L.: 1968, Stars and Stellar Systems 7, 1.

Townes, C. H. and Schawlow, A. L.: 1956, Microwave Spectroscopy, McGraw-Hill, London.

Turner, B. E.: 1970, J. Roy. Astron. Soc. Can. 64, 221.

Verschuur, G. L.: 1971, Astrophys. J. 165, 651.

Wynn-Williams, C. G., Becklin, E. E., and Neugebauer, G.: 1972, Monthly Notices Roy. Astron. Soc. 160, 1.

Zuckerman, B., Yen, J. L., Gottlieb, C. A., and Palmer, P.: 1972, Astrophys. J. 177, 59.

\section{R. D. Davies}

\section{University of Manchester,}

Nuffield Radio Astronomy Laboratories,

Jodrell Bank, Cheshire SK11 9DL, United Kingdom

\section{DISCUSSION}

Turner: Use of the Litvak (1969) IR pumping theory to deduce total particle densities in W3(OH) is in-
appropriate for two reasons:

(i) That theory applies to type II (a) and type II (b) OH masers, whereas the W3(OH) source is a type I source.

(ii) Even if the Litvak (1969) theory did apply, the IR line strengths for the $2.8 \mu \mathrm{m} \mathrm{OH}$ transition have been recently revised downward by a factor of 10 to 100 . This lowers the particle density estimates by a
similar factor, for the same pumping action.

Davies: There is no generally accepted pumping theory for the Type I sources. In my estimates of gas density I have used the conclusion of a number of authors who have examined both collisional and radiative pumping mechanisms. To obtain the observed maser gain an $\mathrm{OH}$ column density of $\sim 3 \times 10^{16} \mathrm{~cm}^{-2}$ is required. This can then be used to derive a gas density and a mass for the cloud.

Robinson: If the $\mathrm{LH}$ and $\mathrm{RH}$ components of the $\mathrm{OH}$ emission are produced by Zeeman splitting, I am puzzled that they are observed to come from spatially separated points in the sky. In the interferometer maps each spot has a characteristic velocity and sense of circular polarization.

Burke: I cannot agree that one should expect to see different Zeeman components from different parts of the sky. On the basis of any theory I know of, the full Zeeman pattern from a given cloud should be
seen if the Zeeman splitting is bigger than the line width.

Davies: The model which discuss in my paper is of an emitting region with small fluctuations in velocity, magnetic field and density. In such a situation you certainly would not expect the LH and RH emission
features to be coincident in apparent position.

Burke: I agree that the general tendency of one hand of circular polarization to be shifted in frequency with respect to the opposite hand is good evidence for the several milligauss field. The main point is that the existing theories fail to explain the absence of the full pattern from each cloud. Some nonlinear theory is
needed, such as the one proposed by Townes. Townes: Presently described theories do not allow for the very large polarizations apparently observed
in a single cloud. A possible way of obtaining such large polarization is to allow for scattering and feedback
by dust particles. Because of the feedback, very by dust particles. Because of the feedback, very large advantage can be given to one polarization over
another, and thus very large polarization

Davies.

splitting where the splitting is comparable with the line expected for masering in the presence of Zeeman will depend upon the particular fluctuations of magnetic fidth. The actual maser gain on any line of sight hand circular polarizations will then have of magnetic field and velocity along that line. Left and right

Zuckerman: The left and right should be com maser gains, as described in my paper.

doublet. You can't wipe out the left in one doublet, wipe out the point if you want to call it a Zeeman another point and then out the left in one doublet, wipe out the right in another doublet coming from

Townes: I don't think that's quite things left over are Zeeman effect. 
field. In other words, it is close enough to have the same magnetic field; it need not come from exactly the same batch of neutral gas.

Burke: I think the principal point is that the evidence the left and right are generally separated is in fact good evidence for a magnetic field there. The fact that you don't see the whole Zeeman pattern is good evidence that existing theory has something seriously wrong, and one must probably have to go to a nonlinear theory such as Townes just described. 